\title{
NON-NUTRITIVE SWEETENERS: EVIDENCE ON THEIR ASSOCIATION WITH METABOLIC DISEASES AND POTENTIAL EFFECTS ON GLUCOSE METABOLISM AND APPETITE
}

\author{
Alonso Romo-Romo ${ }^{1}$, Carlos A. Aguilar-Salinas ${ }^{1}$, Rita A. Gómez-Díaz ${ }^{2}$, Griselda X. Brito-Córdova ${ }^{1}$ \\ Donají V. Gómez-Velasco ${ }^{1}$, María J. López-Rocha ${ }^{1}$ and Paloma Almeda-Valdés ${ }^{1 *}$ \\ ${ }^{1}$ Department of Endocrinology and Metabolism, Instituto Nacional de Ciencias Médicas y Nutrición Salvador Zubirán; \\ ${ }^{2}$ Medical Research Unit in Clinical Epidemiology, UMAE Hospital de Especialidades, Centro Médico Nacional Siglo XXI, \\ Instituto Mexicano del Seguro Social (IMSS). Mexico City, Mexico
}

\begin{abstract}
There is ongoing debate concerning non-nutritive sweeteners, their usage, and their effects on metabolism. The association between non-nutritive sweeteners consumption, development of metabolic diseases, and changes in appetite-regulating hormones is not clear. The aim of this article is to present an overview of non-nutritive sweeteners and to examine the scientific evidence of their effects on glucose metabolism and appetite-regulating hormones. Some observational studies suggest an association between non-nutritive sweeteners consumption and development of metabolic diseases; however, adiposity is a confounder frequently found in these studies. Results of the available clinical trials are heterogeneous and not comparable because of major differences between them. Future controlled studies evaluating specific non-nutritive sweeteners, with an appropriate sample size, including a uniform study group, with sufficient exposure time, and considering adjustment for confounder variables, such as anthropometric characteristics, previous consumption of non-nutritive sweeteners, and coexistence of significant metabolic comorbidities, are needed. (REV INVES CLIN. 2017;69:129-38)
\end{abstract}

Key words: Non-nutritive sweeteners. Diabetes. Metabolic syndrome. Obesity. Appetite hormone.

\section{INTRODUCTION}

By definition, non-nutritive sweeteners (NNS), otherwise known as very low-calorie sweeteners, artificial sweeteners, non-caloric sweeteners, or intense

Corresponding author:

*Paloma Almeda-Valdés

Department of Endocrinology and Metabolism

Instituto Nacional de Ciencias Médicas

y Nutrición Salvador Zubirán

Vasco de Quiroga, 15

Col. Belisario Domínguez Sección XVI, Del. Tlalpan

C.P. 14080, Ciudad de México, México

E-mail: paloma.almedav@incmnsz.mx sweeteners, are substances with a higher intensity of sweetness per gram than caloric sweeteners such as sucrose, corn syrup, and fruit juice concentrate, used in small quantities providing no or few calories $^{1}$.
Received for publication: 10-01-2017

Accepted for publication: 02-03-2017 
At present, there are eight NNS approved to be used as sweeteners in foods and other products, which include sucralose, aspartame, saccharin, acesulfame-K, neotame, advantame, steviol glycosides, and Luo Han Guo fruit extracts. All of these NNS are generally recognized as safe for humans by institutions like the US Food and Drug Administration (FDA), the Joint FAO/ WHO Expert Committee on Food Additives (JECFA), and the European Food Safety Authority (EFSA). The last two NNS mentioned are obtained from natural sources; this is why it is now inappropriate to name all the NNS "artificial sweeteners", as in the past ${ }^{2,3}$.

Although these compounds have very different chemical structures, they all have in common the ability to powerfully activate some of the multiple potential ligand binding sites of the heterodimeric T1R2 + T1R3 sweet-taste receptor in humans ${ }^{4}$.

Consumption of NNS is suggested as a strategy in nutritional therapy to reduce sugar and energy intake in persons with obesity, diabetes, and other metabolic diseases. Their intake has increased in recent years, and currently there is a widespread availability of products containing NNS. In addition, some recent studies have described potential metabolic effects of NNS. Therefore, the objective of this article is to review and analyze the information regarding the association between NNS consumption and the development of metabolic diseases, and to discuss the possible effects of these substances on glucose metabolism and appetite hormones.

\section{OVERVIEW OF NON-NUTRITIVE SWEETENERS}

\section{Saccharin}

Saccharin was the first approved and used NNS in 1879. It has been used as a non-caloric sweetener in foods and beverages for more than 100 years 5 . It is 200-700 times sweeter than table sugar (sucrose) and does not contain any calories. It is not metabolized in the body and is heat stable ${ }^{2}$. Saccharin is eliminated from the body without changes, primarily through urine ${ }^{6}$.

Concerns about saccharin use increased after a study in 1960 showed that saccharin at high levels may cause bladder cancer in laboratory rats, which led to mandate additional studies and a warning label on saccharin-containing products until such a warning could be shown to be unnecessary. Since then, more than 30 human studies have demonstrated that the results found in rats were not relevant for humans, and that saccharin is safe for human consumption. Products containing saccharin are no longer required to have the label warning 5,7 .

\section{Aspartame}

Since aspartame is composed of amino acids, it contains a minimum amount of calories (4 kcal/gram). However, it is about 200 times sweeter than table sugar; therefore, it is consumed in minimal quantities, providing insignificant amounts of energy ${ }^{8}$.

Aspartame is unique among low-calorie sweeteners since it is completely broken down into its constituent amino acids, aspartic acid and phenylalanine, and a small amount of ethanol. These components are found in greater amounts in common foods such as meat, milk, fruits, and vegetables, and are metabolized in the body in the same way, whether they come from aspartame or from foods ${ }^{5}$.

In 1981, the FDA approved aspartame to be used under specific conditions: as a table sweetener, in chewing gum, cold breakfast cereals, and dry bases for some foods (i.e., beverages, instant coffee and tea, jellies, puddings, fillings, dairy products, and toppings) ${ }^{8}$. In 1996, the FDA approved aspartame as a general-purpose sweetener. It is not heat-stable and loses its sweetness when heated, so typically it is not contained in baked goods ${ }^{2,7}$. Labels of aspartame-containing foods and beverages must include a statement that informs that the product contains phenylalanine ${ }^{7,8}$. Nevertheless, long-term studies in both children and adults have shown that aspartame does not alter fasting plasma phenylalanine levels ${ }^{6}$.

\section{Acesulfame potassium}

Acesulfame potassium (Ace-K) is a combination of an organic acid and potassium and is named on the food labels as acesulfame $K$, acesulfame potassium, or Ace- $K^{7}$. It is about 200 times sweeter than sugar and is often combined with other sweeteners ${ }^{2,5}$. 
In 1988, Ace-K was approved by the FDA in non-caloric beverages. Later, in 2003, its general use was approved ${ }^{5}$. It is $100 \%$ absorbed but not metabolized, and excreted within 24 hours. This substance is only $20 \%$ potassium, so even a 12 -ounce diet soda with Ace- $\mathrm{K}$ would merely add $12 \mathrm{mg}$ of potassium to the dietary intake ${ }^{6}$.

Ace- $K$ is heat stable, meaning that it stays sweet even when used at high temperatures during baking, making it suitable as a sugar substitute in baked goods ${ }^{2}$.

\section{Sucralose}

Sucralose is a disaccharide in which three chlorine molecules replace three hydroxyl groups on the sucrose molecule 7 . It is about 600 times sweeter than sugar $^{2}$. It has been shown to have little or no absorption, and it is not metabolized in either human or animal models ${ }^{6}$. The limited metabolism of sucralose occurs directly in tissues and not in the gut lumen?. In 1998, the FDA approved sucralose for use in 15 food categories, and in 1999 for use as a general-purpose sweetener. Sucralose can be found in a variety of foods, including baked goods, beverages, chewing gums, jellies, and frozen dairy desserts. It is heat-stable, making it suitable as a sugar substitute in baked goods ${ }^{10}$.

\section{Neotame}

Neotame is a derivative of dipeptide phenylalanine and aspartic acid ${ }^{7}$. It is approximately 7,000-13,000 times sweeter than table sugar. This substance is rapidly metabolized and completely eliminated from the body, reducing the availability of phenylalanine ${ }^{11}$. In 2002, the FDA approved neotame to be used as a generalpurpose sweetener and as a flavor enhancer in foods, except in meat and poultry. It is heat-stable, making it suitable as a sugar substitute in baked goods ${ }^{2}$.

\section{Stevia}

Stevia is the generic term used for food ingredients derived from the herb Stevia rebaudiana, a plant native to South America, discovered by Bertoni. Steviol glycosides are a more precise term for a group of intensely sweet compounds extracted and purified from the leaves of this plant ${ }^{12}$. Steviol glycosides are NNS and they are 200-400 times sweeter than table sugar. In contrast to the other NNS, stevia has an unusual metabolic route in the body. It is absorbed as steviol after bacterial degradation in the colon, glucuronidated by the liver, and transported back to the intestine where it is metabolized and excreted ${ }^{6}$. The use of purified stevia extracts as sweeteners is currently permitted in a wide number of countries. The FDA has categorized steviol glycosides of high purity ( $95 \%$ minimum purity) as generally recognized as safe. The different types of steviol glycosides include rebaudioside $A$ (also known as Reb A), stevioside, rebaudioside $D$, and steviol glycoside mixture preparations with rebaudioside $A$ and/or stevioside as the predominant components. In contrast, the use of the stevia leaf and crude stevia extracts is not generally recognized as safe, and their use as sweeteners is not permitted ${ }^{12,13}$.

\section{Advantame}

Advantame is the most recently NNS approved by the FDA and the JECFA to be used as a general-purpose sweetener and flavor enhancer. It is structurally related to aspartame, but sweeter (approximately 20,000 times sweeter than sucrose). It is stable to low $\mathrm{pH}$ and high temperature, which indicates that it can be used as a sugar substitute in many products, including baked goods ${ }^{2,14}$.

Due to its high sweetness, advantame is used in very small quantities, does not provide significant amounts of calories, and can be consumed by people with phenylketonuria ${ }^{14,15}$.

\section{Luo Han Guo}

The Siraitia grosvenorii Swingle fruit extracts, usually known as Luo Han Guo or monk fruit, is an extract with sweet taste containing different non-nutritive mogrosides (mostly mogroside $V$ ) obtained from a plant native to Southern China, and it recently received the "generally recognized as safe" status by the FDA2,15.

This sweetener has gained popularity and good acceptance at the same time with stevia because both are NNS derived from natural sources, but the Luo Han Guo has not been commercialized globally yet ${ }^{16}$. It is about 100-250 times sweeter than sucrose, and its acceptable daily intake (ADI) has not been specified yet ${ }^{2}$.

Table 1 summarizes the characteristics of $\mathrm{NNS}^{1,2}$. 
Table 1. Characteristics of the non-nutritive sweeteners

\begin{tabular}{|c|c|c|c|c|}
\hline $\begin{array}{l}\text { Non-nutritive } \\
\text { sweetener }\end{array}$ & $\begin{array}{c}\text { ADI* FDA } \\
\text { (mg/kg body weight) }\end{array}$ & $\begin{array}{c}\text { ADI JECFA } \\
\text { (mg/kg body weight) }\end{array}$ & Year FDA approved & $\begin{array}{l}\text { Times sweeter } \\
\text { than sucrose }\end{array}$ \\
\hline Saccharin & 15 & 5 & 1958 & $200-700$ \\
\hline Aspartame & 50 & 40 & 1981 & 200 \\
\hline Acesulfame-K & 15 & 15 & 1988 & 200 \\
\hline Sucralose & 5 & 15 & 1999 & 600 \\
\hline Neotame & 0.3 & 2 & 2002 & $7,000-13,000$ \\
\hline Stevia & 4 & 4 & 2008 & $200-400$ \\
\hline Luo Han Guo & - & - & 2010 & $100-250$ \\
\hline Advantame & 32.8 & 5 & 2014 & 20,000 \\
\hline
\end{tabular}

*Acceptable daily intake is a measure of the amount of a specific substance in food or drinking water than can be ingested over a lifetime without an appreciable health risk. It is usually expressed in milligrams of sweetener per kilogram of body weight (mg/kg body weight) per day. The amount is usually set at $1 / 100$ of the maximum level at which no adverse effects were observed in animal experiments ${ }^{1}$.

ADI: acceptable daily intake; FDA: U.S. Food and Drug Administration; JECFA: Joint Food and Agriculture Organization and the World Health Organization Expert Committee in Food Additives.

\section{METABOLIC EFFECTS OF NON-NUTRITIVE SWEETENERS: MECHANISMS}

Recently, some investigations have evaluated the effects of NNS on glucose metabolism and appetiteregulating hormones, postulating diverse mechanisms by which NNS may have an influence on glucose, insulin, and other peptide concentrations.

Taste receptors, located in the taste buds cells, trigger the secretion of diverse hormones implicated in the satiety sensation that works as a negative feedback mechanism after food ingestion. These include cholecystokinin, neuropeptide $Y$, peptide YY, glucagon, glucagon-like peptide (GLP) types 1 and 2, ghrelin, oxytocin, galanin, and leptin, among others. It has been observed that taste buds and Langerhans islets have similar phenotypic, structural, and functional characteristics involved in the processing and regulation of nutrient ingestion. In addition, many of the hormones secreted by taste buds in the tongue are expressed by intestinal cells, where they have a key role in food intake and digestion ${ }^{17}$.

In vitro studies have shown that sucralose increases GLP-1 and GLP-2 secretion in mice intestinal L-cells through interaction with taste receptors. Similarly, sucralose, Ace-K, and saccharin stimulate insulin secretion, activating taste receptors in mice pancreatic beta cells. However, these findings have not been replicated in human studies ${ }^{18}$.
Sugar sensing in the intestinal tract alters nutrient absorption and hormone secretion ${ }^{19}$. Margolskee, et al. ${ }^{20}$ showed in an animal model that enteroendocrine cells express T1R2 and T1R3. In their study, glucose and NNS in the intestinal lumen stimulated taste receptors and increased GLP-1 and glucose-dependent insulinotropic peptide (GIP) secretion, promoting expression of SGLT1 and glucose absorption. In addition, Mace, et al. ${ }^{21}$ found that sweet taste receptors in the rat small intestine stimulate glucose absorption through apical GLUT2. They showed that NNS stimulate intestinal T1R2 and T1R3, increasing GLUT2 expression and glucose transport at the luminal intestinal cells. However, these effects have not been demonstrated in humans and the increase on passive and active intestinal glucose transport cannot be considered a conclusive effect of NNS.

Lastly, another postulated mechanism for the metabolic effects of NNS is the alteration of the intestinal microbiome. Data from studies in animal models and from a small study in human subjects suggest that NNS affect gut microbiota. Schiffman, et al. showed that gut microbiota in rats was altered after 12 weeks of exposure to sucralose 22 . The numbers of total anaerobes, bifidobacteria, lactobacilli, Bacteroides, clostridia, and total aerobic bacteria were significantly reduced, without an effect on enterobacteria.

Suez, et al. ${ }^{23}$, in a study performed in rodents, showed that after an 11-week exposure to high doses of saccharin, sucralose, and aspartame, glucose concentrations were significantly augmented. After a four-week 
broad-spectrum antibiotic treatment, the glucose variation was abolished. Subsequently, a feces transplant from saccharin-exposed humans to non-exposed rodents was performed. After the transplant, glucose concentrations showed an increase. The microbiome exhibited a significant imbalance with an increase in the Bacteroides genus and Clostridiales order, suggesting that saccharin modifies intestinal microbiome to the detriment of glucose tolerance.

Palmnäs, et al. showed in rats that after eight weeks of low-dose aspartame consumption, glucose levels increased and insulin-stimulated glucose disposal was impaired. This was associated with an increase in gut total bacteria, Enterobacteriaceae, Clostridium leptum, and Roseburia ssp. In addition, in rats fed with a high-fat diet, aspartame attenuated the increase in the Firmicutes:Bacteroidetes ratio ${ }^{24}$.

The role of gut microbiota in health and disease has been highlighted in recent years. Although the gut microbiota is generally constant over life in adults, it is known that it can be altered importantly by many factors like the use of antibiotics, anti-inflammatory drugs, laxatives, antacids, and chemotherapy. To a lesser degree, gut microbiota can be modified by diet, stress, and consumption of chlorinated water ${ }^{25}$. Furthermore, the impacts of diet and lifestyle on gut microbiota are less well defined, and it could take months to see considerable changes in the microbiome induced by diet ${ }^{26,27}$.

The evidence regarding the relationship between NNS intake and changes in gut microbiota is contradictory. Specific changes in gut microbiota have been associated with diseases like obesity, metabolic syndrome, inflammatory bowel disease, irritable bowel syndrome, diabetes, neurodevelopmental disorders, autoimmune diseases, and allergies ${ }^{28}$. Microbiota dysbiosis occurring in obesity is well described, characterized by an increment of Firmicutes and a decrement of Bacteroidetes ${ }^{26}$. The changes observed in the gut microbiota induced by NNS, until now, do not allow establishing a certain modification. However, this is a new research area in the study of the potential NNS metabolic effects, and it is necessary to continue exploring the relationship between NNS, metabolic alterations, and changes in gut microbiota.

Figure 1 summarizes the postulated mechanisms of NNS metabolic effects.
Figure 1. Potential mechanisms involved in non-nutritive sweeteners' metabolic effects.

Small intestine: In animal models, NNS through stimulation of T1R2 and T1R3 receptors increase secretion of GLP-1 and GIP; these in turn augment the expression of SGLT-1, increasing active glucose transport. Another postulated mechanism is that NNS induce expression of GLUT-2, increasing passive glucose transport.

Colon: Animal studies and a small study in humans demonstrated changes in the gut microbiota induced by NNS associated with effects on glucose metabolism (see text for details).

Pancreas: In vitro studies have postulated that some NNS, through the interaction with taste receptors (T1R2 and T1R3), stimulate insulin release.


\section{SAFETY ASPECTS OF NON-NUTRITIVE SWEETENERS}

The safety of NNS is demonstrated according to a series of studies that include: (i) preliminary in vitro tests with or without metabolic activation demonstrating safety based on the following tests: Amos test, micronucleus test, and cell cultures; (ii) studies of biological absorption, kinetics, excretion, metabolic, and biochemical pathways; (iii) in vivo acute toxicity; (iv) in vivo chronic toxicity; (v) reproductive effects 
in males and females during gestation; (vi) reproductive effects in second and third generations; (vii) special studies; (viii) security limits in population at high risk of extremely high consumption of NNS; (ix) determination of the maximum tolerated dose, meaning the amount that is considered safe and that does not cause alterations when it is consumed daily during a lifetime; and $(x)$ determination of the ADI, which is 100 or more times inferior to the maximum tolerated dose ${ }^{29}$.

Before a NNS is approved for human consumption, in a first stage, studies need to prove that it does not produce cellular mutations, does not cause cellular neoplastic changes or nuclear atypia, does not change membrane composition, and does not modify oxygen consumption and utilization. In a second stage, constant intestinal absorption is verified, and also that its depuration does not alter renal and/or hepatic function, that it does not cause in vitro adverse effects, does not modify energetic and plastic metabolic pathways, and does not produce hydric, electrolyte, acid-base or osmotic changes. In a third stage, it is demonstrated that consumption in healthy volunteers does not cause secondary effects, tissue damage, neoplastic changes or short-, middle- or long-term metabolic modifications. Similarly, it is verified that it does not induce fetal malformations or fertility problems in at least three generations in laboratory animals. Finally, the maximum amount that a human can tolerate without any local or systemic manifestation is determined. Once this maximum amount is known, the ADI is established to be at least 100 or more times inferior to this quantity ${ }^{29}$.

\section{SCIENTIFIC EVIDENCE: OBSERVATIONAL AND CLINICAL TRIALS IN HUMANS}

In recent years, evidence about the NNS provided from clinical trials and observational studies has questioned the absence of metabolic effects of these substances in the body.

More than 10 observational prospective studies have evaluated the association between the consumption of artificially sweetened beverages (ASB) and the development of metabolic diseases like diabetes, metabolic syndrome, or obesity. The majority of these studies concluded that there is a significant association between the consumption of ASB and the risk to develop these metabolic diseases; however, most of these associations were attenuated after adjustment for variables, including age, smoking, physical activity, alcohol consumption, family history of diseases, diet quality, and energy intake. Interestingly, when an additional adjustment for variables related to adiposity, such as body mass index (BMI) and waist circumference, was performed, these associations did not remain statistically significant and the relationship could not be confirmed.

Among these observational studies, the Nurses' Health Study (NHS) I and II followed 74,749 and 91,249 women over eight and 24 years, respectively ${ }^{30,31}$. In the NHS I, the association between the consumption of artificially sweetened carbonated beverages and the incidence of type 2 diabetes (T2D) was significant for both caffeinated and caffeine-free beverages, with a 1.59 relative risk (RR) $(95 \% \mathrm{Cl}: 1.47-1.71$; $\mathrm{p}<0.001)$ and $1.76(95 \% \mathrm{Cl}: 1.63-1.89 ; \mathrm{p}<0.001)$, respectively. After adjustment for several variables, including medical history, lifestyle factors, other concomitant diseases, total energy intake, and BMI using Cox proportional hazards regression models, the association remained significant for caffeine-free beverages but not for caffeinated beverages (RR: 1.09; $95 \% \mathrm{Cl}: 1.00-1.18 ; \mathrm{p}=0.02)$ and (RR: $1.01 ; 95 \% \mathrm{Cl}$ : 0.93-1.10; $p=0.99$ ), respectively ${ }^{30}$.

The NHS II did not find a relationship between the consumption of one or more diet soft drinks per day and the development of diabetes (RR: $1.21 ; 95 \% \mathrm{Cl}$ : $0.97-1.50 ; p=0.12$ ), and this association did not change after an additional adjustment for caloric intake ${ }^{31}$.

The Health Professionals Follow-Up Study (HPFS) followed 40,389 men over 20 years, identifying 2,680 incident cases of T2D during the study. Consumption of ASB was associated with the development of T2D (HR: $1.91 ; 95 \% \mathrm{Cl}: 1.72-2.11 ; \mathrm{p}<0.01$ ); however, this association was not maintained after multivariable adjustment, including total energy intake and BMI (HR: 1.09; 95\% Cl: 0.98-1.21; $\mathrm{p}=0.13)^{32}$.

The European Prospective Investigation into Cancer and Nutrition (EPIC) study is a large cohort that included eight European countries and followed 340,234 adults 
over 16 years. A significant association was found between the consumption of one or more servings per day of ASB and the incidence of T2D (HR: 1.84; $95 \%$ $\mathrm{Cl}: 1.52-2.23 ; \mathrm{p}<0.001)$. After multivariable adjustment, including $\mathrm{BMI}$, the association did not remain statistically significant (HR: $1.13 ; 95 \% \mathrm{Cl}: 0.85-1.52$; $p=0.24)^{33}$.

Greenwood, et al. performed a meta-analysis to evaluate the association between sugar-sweetened and artificially sweetened soft drinks and the incidence of T2D, and found that both types of beverages increased the risk to develop this disease with an RR of 1.20 (95\% Cl: $1.12-1.29 ; \mathrm{p}<0.001)$ and $1.13(95 \% \mathrm{Cl}$ : $1.02-1.25 ; p=0.02$ ), respectively ${ }^{34}$.

Another recent meta-analysis found that sugar-sweetened beverages, ASB, and fruit juices had a positive association with incident T2D. After multivariable adjustment considering adiposity and calibration for information and publication bias, the relationship between ASB and the development of T2D lost significance (RR: 1.22 ; $95 \% \mathrm{Cl}$ : 0.98-1.52; $\mathrm{p}=0.07$ ). However, it was concluded that both ASB and fruit juice ingestion are not advisable as a prevention strategy to develop T2D 35 .

All the observational studies evaluating the relationship between ASB consumption and the incidence of metabolic syndrome have reported significant associations, even after a multivariable adjustment ${ }^{36-38}$. Nevertheless, none of these studies performed an adjustment for variables related to adiposity, except for the Multi-Ethnic Study of Atherosclerosis (MESA), in which the association (HR: 1.36 ; $95 \% \mathrm{Cl}: 1.11$ 1.66; $\mathrm{p}<0.001$ ) lost statistical significance (HR: 1.17; $95 \% \mathrm{Cl}: 0.96-1.44 ; \mathrm{p}=0.06$ ) after adjustment for $\mathrm{BMI}$ and waist circumference ${ }^{39}$.

The relationship between the consumption of beverages containing NNS and obesity development must be explored. To date, only one observational study has been identified assessing this association, and a definite conclusion cannot be established ${ }^{40}$.

Evidence from observational prospective studies indicates that there could be a relation between ASB intake and the development of chronic metabolic diseases. A possible explanation for the attenuation or loss of these associations after adjustment for adiposity is that people that are prone to gain weight tend to consume this kind of beverage as a strategy to lose weight or reduce their energy intake. In this situation, there could be other lifestyle or genetic factors that may impact in the development of these diseases beyond the ingestion of ASB. In addition, subjects with obesity or overweight are at risk to develop metabolic diseases. Another limitation of these studies is that they only measured the consumption of ASB, and these are not the only sources of NNS; therefore, the "non-consumers" could also be exposed to NNS in other products. In table 2 , we show a summary of the observational prospective studies evaluating the association between ASB consumption and T2D or metabolic syndrome development, adjusted by adiposity related variables. The studies that did not find a significant association in the crude risk were not included because no further adjustment was needed.

In a systematic review, we found that there are 28 clinical trials evaluating the effect of different NNS on variables related to glucose metabolism and appetite ${ }^{41}$.

Some clinical trials have reported lower glucose and insulin concentrations after the consumption of diverse NNS; however, in most cases these results are comparing NNS to caloric sweeteners (glucose or sucrose), which are expected to have an impact on glucose and insulin concentrations ${ }^{42-44}$. There are three studies comparing water or placebo to NNS consumption that found lower glucose levels ${ }^{43,45,46}$. Two studies have observed a higher insulinogenic index after the consumption of stevia or aspartame ${ }^{44,45}$. Horwitz, et al. found a higher mean insulin area under the curve (AUC) after aspartame consumption compared to saccharin or an unsweetened beverage ${ }^{47}$.

Pepino, et al. ${ }^{48}$ and Suez, et al. ${ }^{23}$ found in their clinical trials deleterious effects of NNS on glucose metabolism. Pepino, et al. showed higher glucose and insulin levels after sucralose consumption compared to water. Furthermore, they demonstrated a decrement in insulin sensitivity and the insulin clearance rate. However, it is difficult to extrapolate the results of this study because they evaluated an acute exposure to only one NNS (sucralose), and the experiments were made in morbidly obese subjects, most of them women. 
Table 2. Observational prospective studies that adjusted by adiposity the association between the consumption of artificially sweetened beverages and the risk to develop metabolic diseases

\begin{tabular}{|c|c|c|c|c|c|}
\hline Study & Disease & $\begin{array}{l}\text { Follow-up } \\
\text { (years) }\end{array}$ & $\mathrm{n}$ & $\begin{array}{l}\text { Crude risk } \\
(95 \% \mathrm{Cl})\end{array}$ & $\begin{array}{l}\text { Risk adjusted by adiposity } \\
\qquad(95 \% \mathrm{Cl})\end{array}$ \\
\hline$\overline{\text { EPIC }^{33}}$ & T2D & 16 & 340,234 & $1.93(1.47-2.54)$ & $1.13(0.85-1.52)$ \\
\hline EPIC-France ${ }^{62}$ & $\mathrm{~T} 2 \mathrm{D}$ & 14 & 66,118 & $3.50(2.49-4.93)$ & $1.68(1.19-2.39)$ \\
\hline EPIC-Norfolk 63 & $\mathrm{~T} 2 \mathrm{D}$ & 10.8 & 24,653 & $1.70(1.35-2.14)$ & $1.17(0.93-1.48)$ \\
\hline HPFS $^{30}$ & $\mathrm{~T} 2 \mathrm{D}$ & 22 & 39,059 & $1.87(1.65-2.12)$ & $1.06(0.93-1.22)$ \\
\hline HPFS-2 $2^{32}$ & $\mathrm{~T} 2 \mathrm{D}$ & 20 & 40,389 & $1.91(1.72-2.11)$ & $1.09(0.98-1.21)$ \\
\hline $\mathrm{JEFS}^{64}$ & $\mathrm{~T} 2 \mathrm{D}$ & 7 & 2,037 & $1.99(1.33-2.98)$ & $1.70(1.13-2.55)$ \\
\hline MESA $^{39}$ & MS & 7 & 5,011 & $1.31(1.07-1.60)$ & $1.17(0.96-1.44)$ \\
\hline MESA $^{39}$ & $\mathrm{~T} 2 \mathrm{D}$ & 7 & 5,011 & $1.63(1.24-2.13)$ & $1.38(1.04-1.82)$ \\
\hline NHS $\left.\right|^{30}$ & $\mathrm{~T} 2 \mathrm{D}$ & 24 & 74,749 & $1.59(1.47-1.71)$ & $1.01(0.93-1.10)$ \\
\hline
\end{tabular}

The associations shown are between the highest amount of artificially sweetened beverage consumption and the incidence of type 2 diabetes or metabolic syndrome in the study. Associations represented as relative risks, hazard ratios, or odds ratios with their respective $95 \%$ confidence intervals. Adiposity adjustment includes a multivariable adjustment plus adjustment by body mass index and/or waist circumference.

EPIC: the European Prospective Investigation into Cancer and Nutrition study; HPFS: the Health Professionals Follow-up Study; JEFS: Japan Employee Factory Study; MESA: the Multi-Ethnic Study of Atherosclerosis; MS: metabolic syndrome; NHS: the Nurses' Health Study;

T2D: type 2 diabetes; ( $n$ ): number of subjects followed in the study.

Suez, et al. performed a small study in humans after different experiments in rodents, observing that saccharin had a strong impact on glucose levels. During seven days, seven subjects (with characteristics not specified) consumed $100 \%$ of the ADI of saccharin and four of the participants developed a significant increase in glucose concentrations. A microbiota transplantation to mice was performed from the saccharin-responder subjects and seven days after the transplant, a high glucose response was also observed in rodents.

The results of this study have been questioned for many reasons: reduced sample size, absence of a control group, insufficient description of the procedures, the inclusion criteria, and the characteristics of the participants. Furthermore, it is important to mention that the effects of NNS on glucose concentrations have been evaluated, and these results have not been previously shown.

While it is possible that the ingestion of NNS has an impact on glucose tolerance and other metabolic aspects, we consider that the current evidence is not conclusive and there is a need to reevaluate the safety of these substances with well-designed, controlled clinical trials.

It is not possible to perform a statistical analysis of the effects observed in the different clinical trials because there is high heterogeneity in their methodologies, included population, outcome variables, and results presentation.

In relation to appetite, studies have used visual analogue scales to measure subjective ratings of variables related to appetite, including hunger, fullness, satiety, and desire to eat, among others ${ }^{44,49-54}$. Another strategy used is to measure ad libitum energy intake from a buffet after the ingestion of NNS $44,52,55,56$. None of these studies could confirm any effect of NNS on appetite, except for one recently published trial that found significantly higher ad libitum intake $(p=0.01)$ after the ingestion of beverages containing aspartame, monk fruit, and stevia compared to sucrose ${ }^{57}$. In this randomized crossover study, Tey, et al. observed that the energy "saved" with the consumption of NNS was compensated at subsequent meals, with no differences in total daily energy intake between the caloric sweetener and the NNS studied ${ }^{57}$.

Some appetite-regulating hormones, including GLP-1, GIP, PYY, glucagon, ghrelin, and cholecystokinin, have been measured in clinical trials in order to investigate if NNS have an impact on their concentrations. In accordance with some in vitro studies, sucralose increased GLP-1 concentration in two trials performed on healthy subjects and in subjects with type 1 diabetes $^{46,58}$. Hall, et al. found lower GLP-1 concentrations after the consumption of aspartame ${ }^{49}$; however, 
Sylvetsky, et al. found that diet sodas containing NNS slightly increased GLP-1 responses to glucose in normal weight, overweight, and obese individuals ${ }^{59}$. Additional effects in other appetite-related peptides have not been found.

It has been described that the ingestion of NNS may dissociate sweetness from energy, disturbing the balance between taste response, appetite, and food preferences, with this problem being more important in children ${ }^{60}$. If the preference for sweet taste is encouraged by consuming NNS, it could promote the consumption of other sweetened and energy-dense foods, increasing energy intake and weight gain. In addition, studies in rodents support that long-term exposure to NNS debilitates cephalic responses activated by sweet taste ${ }^{4}$. The NNS have been associated with a variety of central and peripheral metabolic consequences, including stimulation of oral and extra-oral sweet taste receptors, alterations in gut microbiota, and impaired ability of sweet taste to predict energy availability, although the mechanisms are still poorly understood 61 .

\section{CONCLUSIONS}

The consumption of NNS can be recommended in specific conditions, for example, in people with obesity and diabetes in whom glycemic and weight control are fundamental. The American Diabetes Association (ADA) and the American Heart Association (AHA) suggest that NNS can be useful in a structured diet to replace sources of added sugars, thereby promoting both energy and carbohydrate intake reduction ${ }^{1}$. The consumption of foods and beverages containing NNS should not encourage a compensatory increase of energy intake from other sources or affect diet quality. For this reason, it is necessary that the consumption of NNS be tied to a healthy lifestyle according to the recommendations of the health professionals, and also to ingest less than the established ADI for each NNS by the FDA and the JECFA.

It is recommendable to avoid both NNS and caloric sweeteners to elude the preference for sweet taste and the consumption of sweet foods that in most cases provide significant amounts of additives and energy from other nutrients like fats. Furthermore, in several circumstances, the consumption of ASB is accompanied by fast foods or other types of nonhealthy foods.

We consider that well-founded conclusions regarding the effect of NNS on metabolism and appetite hormones cannot be proven, and there is a need for additional controlled studies evaluating each NNS, with a proper sample size, including a uniform study group, with a sufficient exposure time, and adjusting for confounder variables such as anthropometric characteristics, previous consumption of NNS, and coexistence of significant metabolic comorbidities.

\section{REFERENCES}

1. Gardner C, Wylie-Rosett J, Gidding SS, et al. Nonnutritive sweeteners: current use and health perspectives: a scientific statement from the American Heart Association and the American Diabetes Association. Diabetes Care. 2012;35:1798-808.

2. US Food and Drug Administration. Food Additives \& Ingredients - Additional Information about High-Intensity Sweeteners Permitted for use in Food in the United States [Internet]. Center for Food Safety and Applied Nutrition. Available at: http:// www.fda.gov/Food/IngredientsPackagingLabeling/FoodAdditi vesIngredients/ucm397725.htm [Accessed 2016 Jan 29].

3. European Food Safety Authority. Sweeteners [Internet]. EFSA 2013. Available at: http://www.efsa.europa.eu/en/topics/to pic/sweeteners [Accessed 2016 Jan 29].

4. Pepino MY. Metabolic effects of non-nutritive sweeteners. Physiol Behav. 2015;152:450-5.

5. Tandel KR. Sugar substitutes: Health controversy over perceived benefits. J Pharmacol Pharmacother. 2011:2:236-43.

6. Magnuson BA, Carakostas MC, Moore NH, Poulos SP, Renwick AG. Biological fate of low-calorie sweeteners. Nutr Rev. 2016; 74:670-89.

7. Fitch C, Keim KS. Position of the Academy of Nutrition and Dietetics: use of nutritive and nonnutritive sweeteners. J Acad Nutr Diet. 2012;112:739-58

8. Shankar P, Ahuja S, Sriram K. Non-nutritive sweeteners: review and update. Nutrition. 2013;29:1293-9.

9. Roberts A, Renwick AG, Sims J, Snodin DJ. Sucralose metabolism and pharmacokinetics in man. Food Chem Toxicol. 2000; 38:S31-41.

10. Qurrat-ul-Ain, Khan SA. Artificial sweeteners: safe or unsafe? J Pak Med Assoc. 2015;65:225-7.

11. Chattopadhyay S, Raychaudhuri U, Chakraborty R. Artificial sweeteners - a review. J Food Sci Technol. 2014;51:611-21.

12. Carakostas MC, Curry LL, Boileau AC, Brusick DJ. Overview: the history, technical function and safety of rebaudioside $A$, a naturally occurring steviol glycoside, for use in food and beverages. Food Chem Toxicol. 2008;46(Suppl 7):S1-10.

13. Urban JD, Carakostas MC, Taylor SL. Steviol glycoside safety: are highly purified steviol glycoside sweeteners food allergens? Food Chem Toxicol. 2015;75:71-8.

14. Warrington S, Lee C, Otabe A, et al. Acute and multiple-dose studies to determine the safety, tolerability, and pharmacokinetic profile of advantame in healthy volunteers. Food Chem Toxicol. 2011;49:S77-83.

15. Sharma A, Amarnath S, Thulasimani M, Ramaswamy S. Artificial sweeteners as a sugar substitute: Are they really safe? Indian J Pharmacol. 2016;48:237-40.

16. Li XE, Lopetcharat K, Drake MA. Parents' and children's acceptance of skim chocolate milks sweetened by monk fruit and stevia leaf extracts. J Food Sci. 2015;80:S1083-92.

17. Calvo SS-C, Egan JM. The endocrinology of taste receptors. Nat Rev Endocrinol. 2015;11:213-27.

18. Jang H-J, Kokrashvili Z, Theodorakis MJ, et al. Gut-expressed gustducin and taste receptors regulate secretion of glucagonlike peptide-1. Proc Natl Acad Sci USA. 2007;104:15069-74.

19. Raybould HE. Sensing of glucose in the gastrointestinal tract. Auton Neurosci. 2007;133:86-90. 
20. Margolskee RF, Dyer J, Kokrashvili Z, et al. T1R3 and gustducin in gut sense sugars to regulate expression of $\mathrm{Na}+$-glucose cotransporter 1. Proc Natl Acad Sci USA. 2007;104:15075-80.

21. Mace OJ, Affleck J, Patel N, Kellett GL. Sweet taste receptors in rat small intestine stimulate glucose absorption through apical GLUT2. J Physiol. 2007;582:379-92.

22. Abou-Donia MB, El-Masry EM, Abdel-Rahman AA, McLendon RE, Schiffman SS. Splenda alters gut microflora and increases intestinal p-glycoprotein and cytochrome p-450 in male rats. J Toxicol Environ Health A. 2008;71:1415-29.

23. Suez J, Korem T, Zeevi $D$, et al. Artificial sweeteners induce glucose intolerance by altering the gut microbiota. Nature. 2014; 514:181-6.

24. Palmnäs MSA, Cowan TE, Bomhof MR, et al. Low-dose aspartame consumption differentially affects gut microbiota-host metabolic interactions in the diet-induced obese rat. PLoS One. 2014;9:e109841.

25. Morales P, Brignardello J, Gotteland M. [The association of intestinal microbiota with obesity]. Rev Med Chil. 2010;138: 1020-7.

26. Conlon M, Bird A. The impact of diet and lifestyle on gut microbiota and human health. Nutrients. 2014;7:17-44.

27. Icaza-Chávez ME. [Gut microbiota in health and disease]. Rev Gastroenterol Mex. 2013;78:240-8.

28. Valdovinos-Díaz MÁ. [Intestinal microbiota in digestive disorders. Probiotics, prebiotics and symbiotics]. Rev Gastroenterol Mex. 2013;78:25-7.

29. Renwick AG. Acceptable daily intake and the regulation of intense sweeteners. Food Addit Contam. 1990;7:463-75.

30. Bhupathiraju SN, Pan A, Malik VS, et al. Caffeinated and caffeine-free beverages and risk of type 2 diabetes. Am J Clin Nutr. 2013:97:155-66.

31. Schulze MB, Manson JE, Ludwig DS, et al. Sugar-sweetened beverages, weight gain, and incidence of type 2 diabetes in young and middle-aged women. JAMA. 2004:292:927-34.

32. de Koning L, Malik VS, Rimm EB, Willett WC, Hu FB. Sugarsweetened and artificially sweetened beverage consumption and risk of type 2 diabetes in men. Am J Clin Nutr. 2011;93:1321-7.

33. Romaguera $D$, Norat $T$, Wark PA, et al. Consumption of sweet beverages and type 2 diabetes incidence in European adults: results from EPIC-InterAct. Diabetologia. 2013;56:1520-30.

34. Greenwood DC, Threapleton DE, Evans CEL, et al. Association between sugar-sweetened and artificially sweetened soft drinks and type 2 diabetes: systematic review and dose-response metaanalysis of prospective studies. Br J Nutr. 2014;112:725-34.

35. Imamura F, O'Connor L, Ye Z, et al. Consumption of sugar sweetened beverages, artificially sweetened beverages, and fruit juice and incidence of type 2 diabetes: systematic review, meta-analysis, and estimation of population attributable fraction. BMJ. 2015;351:h3576.

36. Dhingra R, Sullivan L, Jacques PF, et al. Soft drink consumption and risk of developing cardiometabolic risk factors and the metabolic syndrome in middle-aged adults in the community. Circulation. 2007;116:480-8

37. Lutsey PL, Steffen LM, Stevens J. Dietary intake and the development of the metabolic syndrome: the Atherosclerosis Risk in Communities study. Circulation. 2008;117:754-61.

38. Duffey KJ, Steffen LM, Van Horn L, Jacobs DR, Popkin BM. Dietary patterns matter: diet beverages and cardiometabolic risks in the longitudinal Coronary Artery Risk Development in Young Adults (CARDIA) Study. Am J Clin Nutr. 2012;95:909-15.

39. Nettleton JA, Lutsey PL, Wang Y, Lima JA, Michos ED, Jacobs DR. Diet soda intake and risk of incident metabolic syndrome and type 2 diabetes in the Multi-Ethnic Study of Atherosclerosis (MESA). Diabetes Care. 2009;32:688-94.

40. Fowler SP, Williams K, Resendez RG, Hunt KJ, Hazuda HP, Stern MP. Fueling the obesity epidemic? Artificially sweetened beverage use and long-term weight gain. Obesity (Silver Spring). 2008; 16:1894-900.

41. Romo-Romo A, Aguilar-Salinas CA, Brito-Córdova GX, Gómez Díaz RA, Vilchis Valentín D, Almeda-Valdes P. Effects of the nonnutritive sweeteners on glucose metabolism and appetite regulating hormones: Systematic review of observational prospective studies and clinical trials. PLoS One. 2016:11:e0161264.

42. Okuno G, Kawakami F, Tako H, et al. Glucose tolerance, blood lipid, insulin and glucagon concentration after single or continuous administration of aspartame in diabetics. Diabetes Res Clin Pract. 1986;2:23-7.
43. Härtel B, Graubaum H, Schneider B, Bier A. The influence of sweetener solutions on the secretion of insulin and blood glucose level. Ernährungsunschau. 1993;40:152-5.

44. Anton SD, Martin CK, Han H, et al. Effects of stevia, aspartame and sucrose on food intake, satiety, and postprandial glucose and insulin levels. Appetite. 2010;55:37-43.

45. Gregersen S, Jeppesen PB, Holst J], Hermansen K. Antihyperglycemic effects of stevioside in type 2 diabetic subjects. Metabolism. 2004;53:73-6.

46. Temizkan S, Deyneli O, Yasar M, et al. Sucralose enhances GLP1 release and lowers blood glucose in the presence of carbohydrate in healthy subjects but not in patients with type 2 diabetes. Eur J Clin Nutr. 2015;69:162-6.

47. Horwitz DL, McLane M, Kobe P. Response to single dose of aspartame or saccharin by NIDDM patients. Diabetes Care. 1988 $11: 230-4$

48. Pepino MY, Tiemann CD, Patterson BW, Wice BM, Klein S. Sucralose affects glycemic and hormonal responses to an ora glucose load. Diabetes Care. 2013;36:2530-5.

49. Hall WL, Millward DJ, Rogers PJ, Morgan LM. Physiological mechanisms mediating aspartame-induced satiety. Physiol Behav. 2003;78:557-62

50. Ford HE, Peters V, Martin NM, et al. Effects of oral ingestion of sucralose on gut hormone response and appetite in healthy normal-weight subjects. Eur J Clin Nutr. 2011:65:508-13.

51. Steinert RE, Frey F, Töpfer A, Drewe J, Beglinger C. Effects of carbohydrate sugars and artificial sweeteners on appetite and the secretion of gastrointestinal satiety peptides. $\mathrm{Br}$ J Nutr. 2011;105:1320-8

52. Maersk M, Belza A, Holst Jj, et al. Satiety scores and satiety hormone response after sucrose-sweetened soft drink compared with isocaloric semi-skimmed milk and with non-caloric soft drink: a controlled trial. Eur J Clin Nutr. 2012;66:523-9.

53. Brown AW, Bohan Brown MM, Onken KL, Beitz DC. Short-term consumption of sucralose, a nonnutritive sweetener, is similar to water with regard to select markers of hunger signaling and short-term glucose homeostasis in women. Nutr Res. 2011; 31:882-8.

54. Bryant CE, Wasse LK, Astbury N, Nandra G, McLaughlin JT. Nonnutritive sweeteners: no class effect on the glycaemic or appetite responses to ingested glucose. Eur J Clin Nutr. 2014;68 629-31.

55. Rodin J. Comparative effects of fructose, aspartame, glucose, and water preloads on calorie and macronutrient intake. Am J Clin Nutr. 1990:51:428-35.

56. Melanson KJ, Westerterp-Plantenga MS, Campfield LA, Saris $\mathrm{WH}$. Blood glucose and meal patterns in time-blinded males, after aspartame, carbohydrate, and fat consumption, in relation to sweetness perception. Br J Nutr. 1999:82:437-46.

57. Tey SL, Salleh NB, Henry J, Forde CG. Effects of aspartame-, monk fruit-, stevia- and sucrose-sweetened beverages on postprandial glucose, insulin and energy intake. Int J Obes. 2017; [Epub ahead of print].

58. Brown RJ, Walter M, Rother KI. Effects of diet soda on gut hormones in youths with diabetes. Diabetes Care. 2012;35:959-64

59. Sylvetsky AC, Brown RJ, Blau JE, Walter M, Rother KI. Hormonal responses to non-nutritive sweeteners in water and diet soda. Nutr Metab (Lond). 2016;13:71.

60. Drewnowski A, Mennella JA, Johnson SL, Bellisle F. Sweetness and food preference. J Nutr. 2012:142:1142-8S.

61. Burke MV, Small DM. Physiological mechanisms by which nonnutritive sweeteners may impact body weight and metabolism. Physiol Behav. 2015:152:381-8.

62. Fagherazzi G, Vilier A, Saes Sartorelli D, Lajous M, Balkau B, Clavel-Chapelon F. Consumption of artificially and sugar-sweetened beverages and incident type 2 diabetes in the Etude Epidemiologique aupres des femmes de la Mutuelle Generale de l'Education Nationale-European Prospective Investigation into Cancer and Nutrition cohort. Am J Clin Nutr. 2013;97:517-23.

63. O'Connor L, Imamura F, Lentjes MA, Khaw KT, Wareham NJ, Forouhi NG. Prospective associations and population impact of sweet beverage intake and type 2 diabetes, and effects of substitutions with alternative beverages. Diabetologia. 2015;58: 1474-83.

64. Sakurai M, Nakamura K, Miura K, et al. Sugar-sweetened beverage and diet soda consumption and the 7-year risk for type 2 diabetes mellitus in middle-aged Japanese men. Eur J Nutr. 2014; 53:251-8. 